\title{
Excitation Spectrum of Andreev Billiards with a Mixed Phase Space
}

\author{
H. Schomerus and C. W. J. Beenakker \\ Instituut-Lorentz, Universiteit Leiden, P.O. Box 9506, 2300 RA Leiden, The Netherlands
}

(Received 21 October 1998)

\begin{abstract}
We present a semiclassical theory for the excitation spectrum of a ballistic quantum dot weakly coupled to a superconductor, for the generic situation that the classical motion gives rise to a phase space containing islands of regularity in a chaotic sea. The density of low-energy excitations is determined by quantum energy scales that are related in a simple way to the morphology of the mixed phase space. An exact quantum mechanical computation for the annular billiard shows good agreement with the semiclassical predictions, in particular for the reduction of the excitation gap when the coupling to the regular regions is maximal. [S0031-9007(99)08840-7]
\end{abstract}

PACS numbers: 74.50.+r, 03.65.Sq, 05.45.-a, 74.80.Fp

The spectral statistics of quantum systems is intimately related to the nature of the corresponding classical dynamics [1]. Two celebrated examples are that chaoticity of the classical dynamics is reflected in the quantum realm by level repulsion while integrability causes level clustering [2]. Recently, confined two-dimensional electron gases (quantum dots) coupled to a superconductor via a ballistic point contact have become a new arena for the study of quantum-classical correspondences [3-6]. Such systems are commonly called Andreev billiards [7], because of the alternation of ballistic motion (as in a conventional billiard) with Andreev reflection [8] at the interface with the superconductor. The proximity of the superconductor causes a depletion of excited states at low energies (proximity effect). It was found [3] that a chaotic Andreev billiard has an excitation gap of the order of the Thouless energy, while an integrable Andreev billiard has no true gap but an approximately linearly vanishing density of states. (The Thouless energy $E_{T}=g \delta / 4 \pi$ is the product of the point contact conductance $g$, in units of $2 e^{2} / h$, and the mean level spacing $\delta$ of the isolated billiard [9].)

Both chaotic and integrable dynamics are atypical. The generic situation is a mixed phase space, with "islands" of regularity separated from chaotic "seas" by impenetrable dynamical barriers. A generally applicable theory for the proximity effect in ballistic systems should address the case of a mixed phase space. In this paper we present such a theory.

In a semiclassical approach we link the excitation spectrum quantitatively and qualitatively to the morphology of noncommunicating regions in phase space. Different regions exhibit greatly varying length scales, which also depend sensitively on the position of the point contact. Still, we find that a general relation exists (in terms of effective Thouless energies) between these classical length scales and the corresponding quantum energy scales. The results for integrable and fully chaotic motion are recovered as special cases. For the mixed phase space our main finding is a reduction of the excitation gap below the value $E_{T}$ of fully chaotic systems. The reduction can be an order of magnitude, as we illustrate by a numerical calculation in the annular billiard [10] shown in Fig. 1.

We consider a two-dimensional ballistic region (a "billiard") of area $A$ (mean level spacing $\delta=2 \pi \hbar^{2} / m A$ ) that is connected to a superconductor by an opening of width $W$ (corresponding to a dimensionless conductance $g=$ $2 W / \lambda_{F}$, where $\lambda_{F}$ is the Fermi wavelength). Classical trajectories consist of straight lines inside the billiard, with specular reflections at the boundaries and retro-reflections (= Andreev reflections) at the interface with the superconductor. We assume that $\delta \ll E_{T} \ll \Delta$, where $\Delta$ is the excitation gap in the bulk superconductor. The first condition, $\delta \ll E_{T}$ or $W \gg \lambda_{F}$, is required for a semiclassical treatment. The second condition, $E_{T} \ll \Delta$, ensures that the excitation spectrum becomes independent of the properties of the superconductor.

The Andreev billiard has a discrete spectrum for $\varepsilon<$ $\Delta$. (The excitation energy $\varepsilon>0$ is measured with respect to the Fermi energy. We count each spin-degenerate level

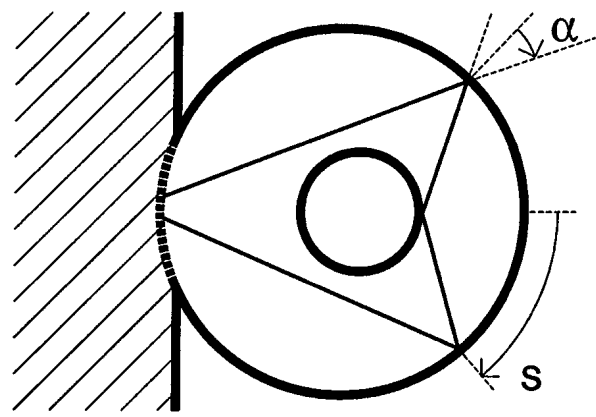

FIG. 1. Andreev billiard consisting of a confined normal conducting region interfacing with a superconductor (shaded) over a distance $W$. The normal region is shaped like an annular billiard, bounded by two excentric circles (outer radius $R$, inner radius $r$, distance of origins $\rho$ ). This figure represents the case $R=1, r=0.35, \rho=0.1, W=0.8$. A periodic trajectory is indicated, involving two Andreev reflections at the interface. For the Poincare map one monitors the collisions with the outer boundary (angle of incidence $\alpha$ and coordinate $s$ along the boundary, with $\alpha=0$ denoting normal incidence and $s=0$ denoting the point closest to the inner circle). 
once.) For $\varepsilon \ll \Delta$ the semiclassical expression for the density of states $\rho(\varepsilon)$ reads [3]

$$
\rho(\varepsilon)=\frac{2}{\delta} \int_{0}^{\infty} d L P(L) \sum_{n=0}^{\infty} \delta\left(\frac{\varepsilon}{2 \pi E_{T}}-\left(n+\frac{1}{2}\right) \frac{L_{T}}{L}\right),
$$

with $P(L)$ the distribution of path lengths between subsequent Andreev reflections. The distribution $P(L)$ is normalized to unity and based on the measure $d s d \sin \alpha$ of initial conditions at the interface with the superconductor (see Fig. 1). The length scale $L_{T} \equiv \hbar v_{F} / 2 E_{T}$ (with $v_{F}$ the Fermi velocity) is determined by the geometry of the billiard by $L_{T}=\pi A / W$ and is therefore purely classical.

Equation (1) was derived in Ref. [3] from the BohrSommerfeld quantization rule. An alternative derivation starts from the Eilenberger equation [11] for the quasiclassical Green function and arrives at an expression (due to Lodder and Nazarov [5]) for the local density of states $\rho(\mathbf{r}, \varepsilon)$ at position $\mathbf{r}$ in the billiard,

$$
\begin{aligned}
\rho(\mathbf{r}, \varepsilon)= & \frac{m}{2 \pi \hbar^{2}} \int_{0}^{2 \pi} d \phi \\
& \times \sum_{n=0}^{\infty} \delta\left(\frac{\varepsilon L(\mathbf{r}, \phi)}{\hbar v_{F}}-\left(n+\frac{1}{2}\right) \pi\right) .
\end{aligned}
$$

Here $L(\mathbf{r}, \phi)$ is the path length between subsequent Andreev reflections for the trajectory passing through $\mathbf{r}$ in direction $\phi$. Equation (1) for $\rho(\varepsilon)=\int d \mathbf{r} \rho(\mathbf{r}, \varepsilon)$ follows from Eq. (2) upon integration over the area of the billiard, by introducing coordinates $l$ along the trajectory and $s, \sin \alpha$ where it hits the interface next, and using $d s d \sin \alpha d l=d \mathbf{r} d \phi$. We can also derive Eq. (1) directly from the quantization condition on the scattering matrix [12], following the steps of Ref. [13].

None of these derivations of Eq. (1) relies on the integrability of the classical dynamics. It may be surprising that Bohr-Sommerfeld quantization can be used for nonintegrable dynamics, but this becomes understandable if we consider that all trajectories become periodic because of Andreev reflection. (The Andreev-reflected hole retraces the path of the incident electron.) We will show that Eq. (1) is quite accurate in nonintegrable systems, but we emphasize that it does not have the status of an equation that becomes asymptotically exact in the classical limit. In contrast to conventional billiards, no quantization condition with this status is known for Andreev billiards.

Return probabilities like $P(L)$ and the related decay of classical correlations have been addressed in many studies [14]. In a chaotic billiard, $L_{T}$ is the mean path length and $P(L) \propto \exp \left(-L / L_{T}\right)$ is an exponential distribution. Equation (1) then gives the density of states

$$
\rho(\varepsilon)=\frac{2 x^{2}}{\delta} \frac{\cosh x}{\sinh ^{2} x}, \quad x=\frac{\pi E_{T}}{\varepsilon},
$$

which drops from $2 / \delta$ (the factor of 2 arises because both electron and hole excitations contribute at positive $\varepsilon$ ) to exponentially small values as $\varepsilon$ drops below $\approx 0.5 E_{T}$.
Equation (3) is close to (but not identical with) the exact quantum mechanical result [3], which has $\rho \equiv 0$ for $\varepsilon \leq$ $0.6 E_{T}$. For integrable motion $P(L)$ decays algebraically $\propto L^{-p}$ with $p$ close to 3. Equation (1) then gives $\rho(\varepsilon) \propto$ $\varepsilon^{p-2}$, hence an approximately linearly vanishing density of states. Numerical studies on the circular and rectangular billiard confirm the validity of the semiclassical approach $[3,15]$.

We turn now to mixed dynamics. Equation (1) allows us to regard each noncommunicating region in phase space as a distinct system, to be labeled by an index $i$. It is helpful to rewrite Eq. (1) in terms of an effective level spacing $\delta_{i}$ and Thouless energy $E_{T, i} \equiv \hbar v_{F} / 2 L_{T, i}$ for each of these regions. (This approach extends the BerryRobnik conjecture [16] to open systems.) We decompose $\rho=\sum_{i} \rho_{i}$ into partial densities of states $\rho_{i}$, defined by

$$
\begin{aligned}
\rho_{i}(\varepsilon)= & \frac{2}{\delta_{i}} \int_{0}^{\infty} d L P_{i}(L) \\
& \times \sum_{n=0}^{\infty} \delta\left(\frac{\varepsilon}{2 \pi E_{T, i}}-\left(n+\frac{1}{2}\right) \frac{L_{T, i}}{L}\right) .
\end{aligned}
$$

The distribution $P_{i}(L)$ (still normalized to unity) now pertains to initial conditions (still with measure $d s d \sin \alpha$ ) on the interface with the superconductor that evolve into the $i$ th region of phase space. On the scale $L_{T, i}$, the distribution $P_{i}(L)$ decays exponentially for chaotic parts of phase space while algebraic decay is found for regular regions [17]. In each case the partial density of states $\rho_{i}$ rises to a value $2 / \delta_{i}$ on an energy scale $E_{T, i}$, but while $\rho_{i}$ has an excitation gap for the chaotic regions it rises linearly for the regular regions. Equation (4) applies to those regions that are accessible for a given location of the interface. We call these "connected" regions. The other "disconnected" regions (usually some of the regular islands) do not feel the proximity of the superconductor and give a constant background contribution $\rho_{i}(\varepsilon)=2 / \delta_{i}$ in the semiclassical approximation.

Two phase space measures $O_{i}$ and $V_{i}$ determine the mean length $L_{T, i}=V_{i} / O_{i}$ between Andreev reflections, the effective level spacing $\delta_{i}=(2 \pi \hbar)^{2} / m V_{i}$, and the effective Thouless energy $E_{T, i}=\hbar v_{F} / 2 L_{T, i}=g_{i} \delta_{i} / 4 \pi$, where $g_{i}=O_{i} / \lambda_{F}$ is the effective dimensionless conductance. The first is the area $O_{i}$ that the region overlaps with the superconducting interface on the Poincare map (see Fig. 2). It is a measure of the coupling strength of a region to the superconductor. The second is the volume $V_{i}$ that the region fills out in the full phase space $(\mathbf{r}, \phi)$.

The phase space that is explored from the point contact can again be parametrized by the variables $s, \sin \alpha$ on the interface and the coordinate $l$ along the trajectory. The identification of $L_{T, i}=V_{i} / O_{i}$ as the mean path length in region $i$ is a consequence of $d s d \sin \alpha d l=d \mathbf{r} d \phi$. The mean length of all trajectories $\langle L\rangle \equiv \int d L L P(L)=$ $\sum_{i}^{\prime} O_{i} L_{T, i} / 2 W=\sum_{i}^{\prime} V_{i} / 2 W$ can be used to determine the total phase space volume $V_{\text {con }} \equiv \sum_{i}^{\prime} V_{i}=2 W\langle L\rangle$ that is 


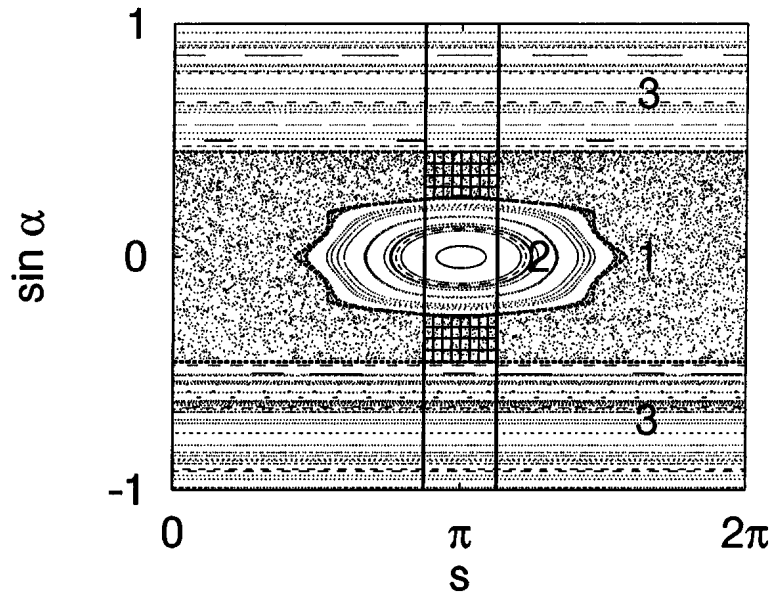

FIG. 2. Poincaré map of the annular billiard of Fig. 1. Dynamical barriers separating regions in phase space are shown as dashed lines. Chaotic trajectories are found in region 1. Region 2 is an island of regular motion around a short stable periodic orbit. Region 3 is integrable and consists of skipping orbits that never hit the inner circle. Initial conditions at the interface with the superconductor are uniformly distributed within the strip around $s=\pi$, of area $2 W$. The hatched area is the overlap $O_{1}$ of this strip with region 1.

connected to the interface with the superconductor. Here the prime denotes restriction of the sum to connected regions, and we used the sum rule $\sum_{i}^{\prime} O_{i}=2 W$. The volume $V_{\text {dis }}$ of the disconnected regions (which determines the background contribution to $\rho$ ) follows from the sum rule $\sum_{i} V_{i}=V_{\text {con }}+V_{\text {dis }}=2 \pi A$.

Since typically the smallest $E_{T, i} \ll E_{T}$, the total density of states $\rho=\sum_{i} \rho_{i}$ has a reduced excitation gap. This is especially the case when one couples maximally to the regular regions. Then their contribution to $\rho$ at small $\varepsilon$ (long path lengths) is minimal (the slope $\propto 1 / E_{T, i} \delta_{i}$ of the linear increase is small since $E_{T, i}$ is large), and the gap is substantially reduced due to long chaotic trajectories. The constant background and the linear increase from regular regions dominates when the coupling is mainly to the chaotic parts of phase space.

The preceding paragraph summarizes the key finding of our work. We illustrate it now for the annular billiard of Fig. 1. The Poincaré map in Fig. 2 shows three main regions [18], one with chaotic motion (1) and two with regular motion ( 2 and 3 ). The regular island 2 corresponds to orbits that bounce back and forth between the two circles where their distance is largest. It has a short stable periodic orbit in its center. Region 3 is integrable and consists of skipping orbits (trajectories that do not hit the inner circle, so that their angular momentum $\sin \alpha$ is conserved).

The regular regions couple maximally to the point contact when it is located at the short stable periodic orbit, as in Fig. 1 (location $s=\pi$ ). We have computed $P(L)$ by following trajectories and obtained $\rho(\varepsilon)$ from Eq. (1). The result is shown in Fig. 3 (solid curve). At the bottom of the spectrum, all discernible features are due to the chaotic region. We see an excitation gap which is

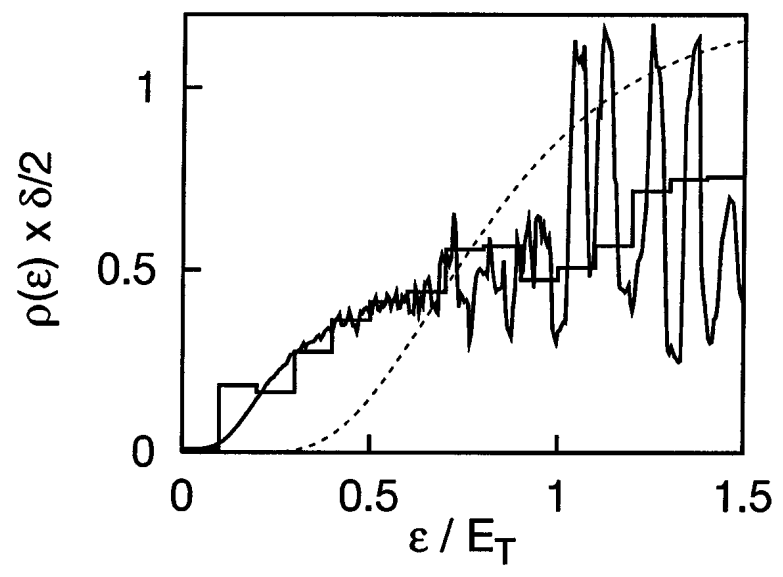

FIG. 3. Density of states of the annular billiard of Fig. 1. The solid curve is the semiclassical prediction computed from Eq. (1). The histogram is obtained by an exact quantum mechanical computation. The dashed curve is the semiclassical result (3) for completely chaotic dynamics.

about a factor of 4 smaller than in the fully chaotic case [Eq. (3), dashed curve]. The reduction originates from long chaotic trajectories with mean length $L_{T, 1} \approx 4 L_{T}$, hence $E_{T, 1} \approx E_{T} / 4$. The linear increase (with slope $\propto$ $\left.1 / E_{T, i}\right)$ of the regular partial density of states is suppressed since for the regular regions $E_{T, i}$ is large. An exact quantum mechanical calculation [19] (histogram) confirms the low- $\varepsilon$ behavior found semiclassically. The sharp peaks at higher $\varepsilon$ in the semiclassical prediction, which arise from families of regular trajectories of almost identical length, are not resolved in the histogram. This is no surprise since their extension in phase space is still less than a Planck cell for numerically accessible Fermi wavelengths. It remains an open question whether these fluctuations would indeed appear with increase of the quantum mechanical resolution.

The regular island is disconnected from the superconductor when the point contact is moved to the other end of the billiard (at $s=0$, where the separation of the circles is smallest). The gap in the chaotic partial density of states is reduced to a lesser degree than before; see Fig. 4(a). Excitations localized in the regular island give a constant background contribution $2 / \delta_{2}=2 m V_{2} /(2 \pi \hbar)^{2}$. If the point contact is placed between these two extreme positions (at $s=1$ ), the regular regions of phase space dominate the low-energy behavior of $\rho(\varepsilon)$. Instead of an excitation gap we observe a smoothly and slowly increasing density of states; see Fig. 4(b). The histograms in Fig. 4 fall systematically below the semiclassical prediction. We attribute this discrepancy to the constant background contribution in the semiclassical result, which should vanish at small $\varepsilon$ because of quantum mechanical tunneling through the dynamical barrier between regions 1 and 2. This source of error is absent in Fig. 3, because there all regions are directly coupled to the superconductor.

In summary, we have found that the superconductor proximity effect in ballistic systems depends sensitively on the morphology of the classical phase space. The 


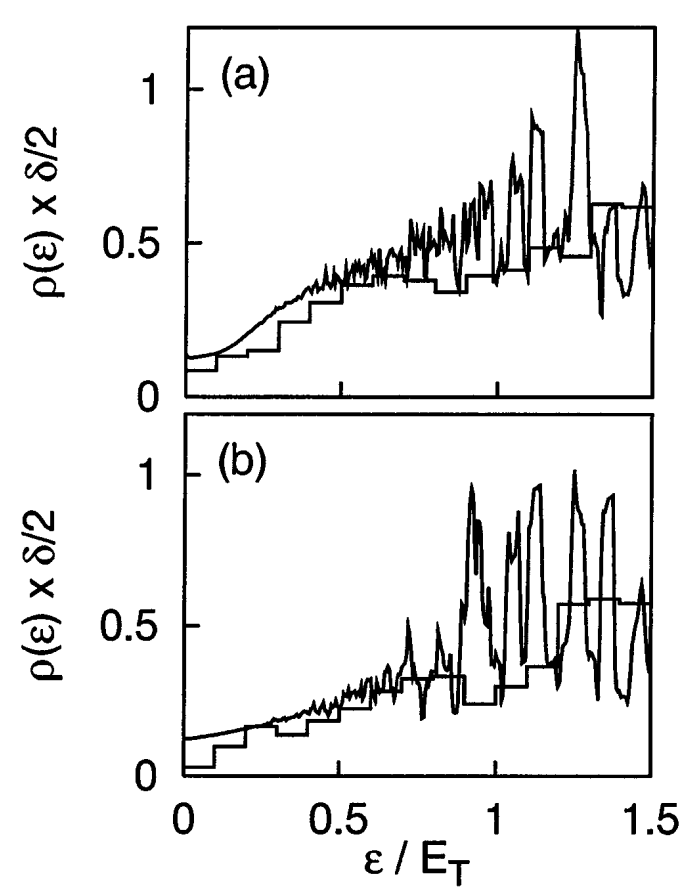

FIG. 4. Density of states of the billiard of Fig. 1, but with two different locations of the interface $[s=0$ in (a) and $s=1$ in (b)]. The semiclassical prediction from Eq. (1) (solid curves) is compared with an exact quantum mechanical computation (histograms).

excitation spectrum at low energies can be described in an intuitively appealing way by means of effective Thouless energies and level spacings for the regular and chaotic regions of phase space. If the coupling to the regular regions is maximal, the excitation spectrum exhibits an excitation gap that is much smaller than the gap of a fully chaotic system. Measurement of such a reduced gap would provide a unique insight into the effect of a mixed classical phase space on superconductivity.

This work was supported by the European Community (Program for the Training and Mobility of Researchers) and by the Dutch Science Foundation NWO/FOM.

[1] M.C. Gutzwiller, Chaos in Classical and Quantum Mechanics (Springer, New York, 1990); F. Haake, Quantum Signatures of Chaos (Springer, Berlin, 1992).

[2] M. V. Berry and M. Tabor, Proc. R. Soc. London A 156, 375 (1977); O. Bohigas, M. J. Giannoni, and C. Schmit, Phys. Rev. Lett. 52, 1 (1984); A. V. Andreev, O. Agam, B.D. Simons, and B.L. Altshuler, Phys. Rev. Lett. 76, 3947 (1996).

[3] J. A. Melsen, P.W. Brouwer, K. M. Frahm, and C.W. J. Beenakker, Europhys. Lett. 35, 7 (1996); Phys. Scr. T69, 223 (1997).

[4] A. Altland and M. R. Zirnbauer, Phys. Rev. Lett. 76, 3420
(1996); K. M. Frahm, P.W. Brouwer, J. A. Melsen, and C. W. J. Beenakker, Phys. Rev. Lett. 76, 2981 (1996).

[5] A. Lodder and Yu. V. Nazarov, Phys. Rev. B 58, 5783 (1998).

[6] A. Altland, B. D. Simons, and D. Taras-Semchuk, Pis'ma Zh. Eksp. Teor. Fiz. 67, 21 (1998) [JETP Lett. 67, 22 (1998)].

[7] I. Kosztin, D. L. Maslov, and P. M. Goldbart, Phys. Rev. Lett. 75, 1735 (1995).

[8] A. F. Andreev, Zh. Eksp. Teor. Fiz. 46, 1823 (1964) [Sov. Phys. JETP 19, 1228 (1964)].

[9] The energy scale $E_{T}$ is the reciprocal mean time between Andreev reflections. We call this energy scale "Thouless energy" by analogy to diffusive systems. Other definitions of the Thouless energy of a ballistic system appear in different contexts. See A. Altland, Y. Gefen, and G. Montambaux, Phys. Rev. Lett. 76, 1130 (1996). (Our $E_{T}$ corresponds to $E_{4}$ in that paper.)

[10] O. Bohigas, D. Boosé, R. Egydio de Carvalho, and V. Marvulle, Nucl. Phys. A560, 197 (1993); E. Doron and S. D. Frischat, Phys. Rev. Lett. 75, 3661 (1995).

[11] G. Eilenberger, Z. Phys. 214, 195 (1968).

[12] C. W. J. Beenakker, Phys. Rev. Lett. 67, 3836 (1991).

[13] E. Doron and U. Smilansky, Nonlinearity 5, 1055 (1992).

[14] F. Vivaldi, G. Casati, and I. Guarneri, Phys. Rev. Lett. 51, 727 (1983); C.F.F. Karney, Physica (Amsterdam) 8D, 360 (1983); B. V. Chirikov and D. L. Shepelyansky, Physica (Amsterdam) 13D, 395 (1984); J.D. Meiss and E. Ott, Physica (Amsterdam) 20D, 387 (1986); W. Bauer and G.F. Bertsch, Phys. Rev. Lett. 65, 2213 (1990); A. Pikovsky, J. Phys. A 25, L477 (1992); H. Alt, H.-D. Gräf, H. L. Harney, R. Hofferbert, H. Rehfeld, A. Richter, and P. Schardt, Phys. Rev. E 53, 2217 (1996).

[15] Discrepancies between exact numerical and semiclassical results for the rectangular billiard found in Ref. [3] are actually within statistical error bars. We redid the calculation for a greatly extended ensemble of billiards (variations in Fermi wavelength at fixed shape) and found good agreement.

[16] M. V. Berry and M. Robnik, J. Phys. A 17, 2413 (1984).

[17] One finds algebraic tails in $P_{i}(L)$ also for chaotic parts of phase space, due to trapping of orbits in the vicinity of borders to regular regions [14]. Since these tails appear only for lengths $L \gg L_{T, i}$, they are irrelevant for the present study.

[18] The chaotic region can be subdivided further into three parts separated by weakly impenetrable dynamical barriers (penetration time larger than $L_{T} / v_{F}$ ). The qualitative description is improved if one assigns to each part its own set of effective quantities $E_{T, i}, \delta_{i}$.

[19] The histograms were obtained by averaging over 250 independent exact spectra (variation of Fermi energy $E_{F}$ at fixed shape). To find the spectra we computed the scattering matrices $S\left(E_{F} \pm \varepsilon\right)$ in incremental steps of $\varepsilon$ much smaller than a mean level spacing. An excitation is encountered when an eigenvalue of $S\left(E_{F}+\varepsilon\right) S^{*}\left(E_{F}-\right.$ $\varepsilon)$ passes through the point -1 on the unit circle [12]. The dimensionality of the scattering matrix was in the range from 25 to 50 . 